\title{
La línea 12 del Metro \\ Crisis, aprendizaje y cambio institucional
}

\author{
Mexico City's Metro Line 12: Crisis, Learning, and Institutional Change
}

\author{
Carlos Moreno Jaimes*
}

Resumen: Este trabajo analiza la crisis originada a raíz de la suspensión del tramo elevado de la línea 12 del Metro de la Ciudad de México desde la perspectiva del aprendizaje organizacional y el cambio institucional. Se pregunta cuáles son los principales aprendizajes que pueden derivarse de un evento tan complejo y polémico como fue el de la línea 12 y en qué medida dichos aprendizajes pueden propiciar cambios institucionales útiles para lidiar con situaciones parecidas. El argumento central es que las oportunidades de aprendizaje y cambio institucional para un gobierno en situaciones de crisis no son muchas debido a la ausencia de información confiable sobre los hechos ocurridos, sus causas y las responsabilidades de cada uno de los involucrados. Se ofrece una revisión de dos perspectivas analíticas que tratan de explicar en qué medida la aparición de eventos críticos pueden detonar aprendizajes y cambios sustantivos de política pública.

Palabras clave: aprendizaje organizacional, cambio institucional, política pública, crisis de gobierno, México.

Abstract: This article analyzes, from the organization learning and institutional change perspectives, the crisis created by the suspension of Mexico City's Metro line 12. What are the main learnings that could be derived from a complex and controversial event such as the interruption of line 12? To what extent a crisis like this can bring about useful institutional changes to deal with similar situations? I argue that the learning opportunities and institutional changes of a government that undergoes a severe crisis are scarce, mainly because reliable information regarding facts, causes and responsibilities is frequently absent. The article reviews two rival analytical perspectives that try to explain to what extent the emergence of critical events yield learning and substantial policy changes.

Keywords: organizational learning, institutional change, public policy, government crisis, Mexico.

${ }^{*}$ Carlos Moreno Jaimes es profesor-investigador en el Departamento de Estudios Sociopolíticos y Jurídicos del Instituto Tecnológico y de Estudios Superiores de Occidente (ITESo). Periférico Sur Manuel Gómez Morín 8585, Tlaquepaque, Jalisco, 45604, edificio E, primer nivel, oficina 207. Tel: +52 (33) 3669 3434, ext. 3751. Correo-e: cmoreno@iteso.mx. ORCID: 0000-0002-4928-218X. El autor agradece los valiosos comentarios de dos dictaminadores anónimos que sirvieron para mejorar el contenido de este trabajo. Agradece también la contribución de María del Mar Morales en la sistematización de la información.

Artículo recibido el 13 de marzo de 2017 y aceptado para su publicación el 31 de mayo de 2017. 


\section{INTRODUCCIÓN}

-7 ste trabajo analiza el caso de la línea 12 (L12) del Metro de la Ciudad de México desde la perspectiva del aprendizaje organizacional y el cambio institucional. Su punto de partida es la crisis que se desató a raíz de que el Gobierno del Distrito Federal decidió suspender el tramo elevado de la L12 del Metro en marzo de 2014 —apenas nueve meses después de que la obra fue entregada de manera definitiva por el consorcio responsable de su diseño y construcción-, lo cual dio origen a una investigación para clarificar los hechos ocurridos y deslindar las responsabilidades de los actores privados y públicos que estuvieron involucrados en las diversas etapas del proyecto. Como es habitual en eventos de este tipo, la crisis de la L12 se caracterizó por una atención mediática muy intensa y por una guerra de acusaciones mutuas entre los principales actores políticos y burocráticos participantes. Independientemente del escándalo político y mediático de la crisis de la L12, llama la atención que una organización gubernamental con casi 50 ańos de experiencia en suministrar un servicio de transporte colectivo masivo a través de once líneas, no haya sido capaz de enfrentar con éxito la construcción y operación de su decimosegunda línea, suponiendo que, a lo largo de su existencia, el Sistema de Transporte Colectivo Metro (sTC-Metro) tuvo que haber desarrollado un conjunto de rutinas y aprendizajes esenciales para seguir ampliando su red. No es el propósito de este trabajo analizar por qué toda esa experiencia acumulada no parece haber servido de mucho para evitar la crisis de la L12. En cambio, este documento se estructura alrededor de la siguiente pregunta: ¿Cuáles son los principales aprendizajes que pueden derivarse de un evento tan complejo y polémico como fue el de la Línea (Des)Dorada y en qué medida dichos aprendizajes pueden propiciar cambios institucionales que sean útiles para lidiar con situaciones parecidas de mejor manera en el futuro?

Lamentablemente, el argumento central de este trabajo no es muy optimista, pues sostiene que las oportunidades de aprendizaje y cambio institucional para un gobierno en situaciones de crisis no son muchas. Si bien las crisis ofrecen "ventanas de oportunidad" (policy windows) para llevar a cabo reformas institucionales y políticas de largo alcance, sus repercusiones sobre el aprendizaje organizacional no son las más propicias para un cambio efectivo. En el caso particular de la L12 del Metro, las oportunidades de generar aprendizajes para el futuro son todavía más reducidas debido a la ausencia de información confiable sobre los hechos ocurridos, sus causas y las responsabilidades de cada uno de los actores involucrados. En otros términos, el propio proceso a partir del cual se va generando la información esencial sobre el caso parece estar altamente politizado, 
de modo que resulta sumamente complicado derivar aprendizajes significativos para los participantes.

En la siguiente sección expongo algunas perspectivas analíticas sobre el aprendizaje gubernamental, y enfatizo que existen dos visiones divergentes que tratan de explicar en qué medida la aparición de eventos críticos es capaz de detonar aprendizajes organizacionales y cambios sustantivos de política pública. Posteriormente, analizo el caso de la L12 del Metro a la luz de dicha discusión, para luego cerrar con algunas reflexiones finales.

\section{CRISIS, APRENDIZAJE Y CAMBIO: LAS MIRADAS ANALÍTICAS Consideraciones sobre el aprendizaje organizacional}

¿Pueden los gobiernos aprender? Esta pregunta, cuya respuesta pudiera parecer evidente a simple vista, sigue siendo materia de discusión entre los estudiosos de la administración pública y disciplinas similares. Como lo explica Stern (1997), alrededor del concepto de "aprendizaje" abundan interrogantes respecto a cuál es el objeto y el sujeto del aprendizaje, qué factores lo ocasionan, mediante qué criterios podemos distinguir si ciertos cambios institucionales, organizacionales o de política pública son en realidad resultado de aprendizajes genuinos o de otras causas, entre muchas otras. No es el propósito de este trabajo hacer una recapitulación detallada de las contribuciones de la literatura al tema del aprendizaje gubernamental, pero sí presentar algunas visiones respecto a qué consecuencias tiene éste sobre el rumbo de las políticas públicas y qué papel juegan las crisis como detonadores de procesos de aprendizaje gubernamental y cambio institucional.

Uno de los trabajos pioneros en el tema del aprendizaje organizacional, el de Huber (1991), afirma que "una organización aprende si, a través de su procesamiento de información, su rango de comportamientos potenciales se transforma" (Huber, 1991: 89). Por su parte, Kim (1993) sostiene que el aprendizaje implica aumentar la capacidad de una organización para tomar acciones efectivas y puede constatarse cuando dicha organización introduce cambios en las rutinas a través de las cuales realiza las cosas para enfrentar nuevas circunstancias en su entorno. Argyris y Schoen (1978) hacen explícito que el aprendizaje individual no puede extrapolarse al aprendizaje organizacional, aunque afirman que las organizaciones aprenden a través de los individuos que actúan como agentes de aquellas:

Las organizaciones no son simplemente una agrupación de individuos, aunque no hay organizaciones sin que exista tal agrupación. De forma similar, el aprendizaje organizacional no 
es simplemente igual al aprendizaje individual, aunque las organizaciones aprenden únicamente a través de la experiencia y las acciones de los individuos (Kim, 1993: 40). ${ }^{1}$

Sin pretender abundar demasiado en la discusión sobre las diferencias en el aprendizaje que ocurre a esos dos niveles (individual y organizacional), conviene enfatizar un elemento en común: que el aprendizaje está altamente influido por los "modelos mentales" que los miembros individuales logran transmitir al resto de su organización, los cuales son un componente que puede llegar a convertirse en una memoria activa, es decir en una información recuperable a la que la organización decide prestar atención como base de su actuación (Kim, 1993). El aprendizaje puede ocurrir dentro de los límites de un mapa mental determinado o bien más allá de éste, dependiendo de la complejidad del problema que la organización enfrente. A esto se refiere Argyris (2001) al afirmar que existen dos formas principales de aprendizaje: el aprendizaje en un solo circuito (single-loop learning) y el aprendizaje en doble circuito (double-loop learning). El primero ocurre cuando se presenta un desajuste entre las expectativas y los resultados de la acción organizacional y el entorno presiona para que se tomen acciones para resolver tal disparidad. Por lo tanto, el objetivo del aprendizaje en un solo circuito consiste en ajustar los resultados a los objetivos originales sin cuestionar los mecanismos causales subyacentes que dieron origen al problema. En cambio, el aprendizaje en doble circuito implica ir más allá, al poner en tela de juicio los mapas mentales que la organización ha establecido para conceptualizar sus problemas de manera habitual. Al cuestionar de fondo los supuestos que están detrás de las políticas y las metas organizacionales, este segundo tipo de aprendizaje desemboca en la adopción de soluciones innovadoras que se alejan de los supuestos subyacentes con los que una organización enfrenta los problemas.

Estas teorías del aprendizaje organizacional han servido de base para desarrollar la noción más reciente —y aparentemente menos consolidada - del aprendizaje gubernamental (Etheredge, 1981). Para Blindenbacher et al. (2010), por ejemplo, el aprendizaje gubernamental se refiere a un cambio estructurado y consciente en la manera de pensar sobre algún problema de política pública, mismo que implica cambios conductuales entre quienes toman decisiones y entre quienes operan los programas (Blindenbacher et al., 2010: 49). Para entender el significado del aprendizaje gubernamental, es necesario tomar en consideración ciertos rasgos que son intrínsecos al gobierno. Fundamentalmente, es importan-

${ }^{1}$ La traducción del inglés es mía. 
te reconocer que los gobiernos no pueden concebirse como entidades unitarias que funcionan bajo una misma racionalidad (Allison, 1971). Si bien todas las organizaciones gubernamentales deben su creación a algún marco institucional que define sus objetivos, tareas y responsabilidades, su dinámica cotidiana está marcadamente influida por referentes culturales muy diversos que los llevan a priorizar fines diferentes y a veces hasta contradictorios (Crozier y Friedberg, 1990). Las organizaciones del gobierno, por otra parte, son altamente permeables a la lógica de su entorno político. Puesto que las organizaciones del gobierno funcionan bajo las reglas de los regímenes democráticos, los bienes o servicios que proveen no están destinados a un público definido con claridad, sino más bien hacia una pluralidad de públicos que van a evaluar con estándares muy diversos el comportamiento y el desempeño del gobierno. Por si ello fuera poco, los gobiernos presentan características que dificultan enormemente los procesos de aprendizaje. En primer lugar, puesto que los líderes gubernamentales emanan de procesos de competencia electoral, éstos suelen dar una enorme prioridad a los compromisos programáticos adquiridos durante las campañas políticas, es decir que son renuentes a modificar sus plataformas político-electorales por temor a decepcionar las expectativas de los votantes. Este comportamiento inflexible no es, en general, favorable para promover el aprendizaje en los gobiernos. En segundo lugar, la temporalidad acotada de la gestión gubernamental influye en la prioridad que los líderes deciden dar a los diferentes asuntos de política pública, ya que las agendas de gobierno son altamente sensibles a los tiempos electorales (Kingdon, 1995). Lo anterior, además, se ve reforzado por el hecho de que los gobiernos, así como los individuos, toman decisiones bajo condiciones de racionalidad acotada (Simon, 1997), lo cual ocasiona que los actores políticos por lo general limiten su atención a un conjunto muy reducido de asuntos, por lo que las políticas públicas permanecen sin experimentar cambios importantes durante periodos relativamente amplios de tiempo (Baumgartner et al., 2014). Por último, si bien un proceso exitoso de aprendizaje gubernamental suele implicar que se operen transformaciones profundas en el comportamiento habitual de las organizaciones, con frecuencia los líderes políticos se muestran reticentes a realizar cambios drásticos debido, sobre todo, a que no es fácil predecir cómo serán interpretados políticamente los resultados: si como triunfos o como derrotas ante la mirada de aliados u opositores. Por ello, es común observar que los servidores públicos, acostumbrados a trabajar en un ambiente de secrecía y uniformidad, prefieren mantener el statu quo frente al riesgo de introducir cambios cuyos efectos podrían ser costosos políticamente. 
Con todo y las dificultades que enfrentan los gobiernos en sus procesos de aprendizaje, hay una corriente de la literatura que se ha preocupado por estudiar bajo qué condiciones los gobiernos pueden aprender y qué consecuencias tiene eso en la adopción de políticas públicas. La siguiente sección aborda estos asuntos, poniendo un énfasis especial en el papel que juegan los episodios de crisis (como el de la suspensión de la L12 del Metro en la Ciudad de México) en detonar aprendizajes gubernamentales y procesos de cambio organizacional y de política pública.

\section{Dos hipótesis rivales sobre las crisis y el aprendizaje gubernamental}

Uno de los trabajos ya clásicos sobre el tema del aprendizaje en políticas públicas es la teoría de las coaliciones promotoras de Paul Sabatier (1988), quien afirma que el proceso de cambio de una política pública requiere entenderse desde una perspectiva temporal amplia (de una década, por lo menos), tomando como unidades de análisis los "subsistemas de política pública" (es decir las redes de actores privados y públicos cuyos intereses confluyen en alguna política pública) —en lugar de hacerlo alrededor de instituciones gubernamentales específicas- y conceptualizando las políticas como "sistemas de creencias" (belief systems), es decir como un conjunto de valores que algunos actores consideran prioritarios y cuya realización (o negación) esos mismos actores atribuyen a causas determinadas, incluidos a ciertos instrumentos de política pública. Uno de los elementos más importantes del modelo de Sabatier son las "coaliciones promotoras" (advocacy coalitions), las cuales consisten en un conjunto de personas pertenecientes a un subsistema de política pública, que ocupan diversas posiciones (funcionarios electos y nombrados, líderes de grupos de interés, investigadores, entre otros), comparten un mismo sistema de creencias y logran coordinarse con relativa eficacia a través del tiempo. El objetivo fundamental de una coalición promotora es traducir su sistema de creencias en programas concretos de política pública, para lo cual utilizan la información y el análisis con el fin de convencer a otros actores y fortalecer sus posiciones frente a otras coaliciones que, de igual manera, buscan imponer sus propios sistemas de creencias en la hechura de las políticas. El argumento principal de Sabatier es que este proceso de argumentación, deliberación y persuasión —que en gran medida coincide con la visión de Majone (1989) sobre la naturaleza del análisis de políticas públicas - que caracteriza la competencia entre coaliciones promotoras para influir en la hechura de las políticas va creando aprendizaje (policy learning) entre las mismas. ${ }^{2}$ Dicho aprendizaje es una de las princi-

\footnotetext{
${ }^{2} \mathrm{El}$ aprendizaje de políticas se define como "un cambio duradero en el razonamiento o en las intenciones de
} 
pales variables que mejor explica la transformación que experimentan las políticas públicas a lo largo del tiempo, aunque también influyen ciertos factores exógenos, como el cambio en las condiciones económicas y tecnológicas y la aparición de una nueva coalición gobernante. Por último, Sabatier se pregunta cuáles son las condiciones bajo las cuales puede florecer más fácilmente el aprendizaje de políticas no sólo al interior de un mismo sistema de creencias - lo que es comparativamente sencillo, ya que existe una tendencia natural a que los actores de una misma coalición promotora aprendan a fortalecer sus argumentos frente a las críticas de sus adversarios-, sino entre coaliciones opuestas. La primera condición es que exista un conflicto informado para que el debate resulte analíticamente productivo. La segunda es que exista un foro de discusión relativamente apolítico, es decir uno que sirva para que las diferencias en las posiciones fundamentales de cada una de las coaliciones se discutan con apego a normas profesionales.

Como ya comenté en el párrafo anterior, el modelo de Sabatier da un peso importante a eventos exógenos como factores capaces de detonar el cambio en las políticas públicas. Uno de esos eventos son las situaciones de crisis. ¿Qué consecuencias tienen las crisis sobre el aprendizaje y el cambio institucional? Consideremos dos visiones alternativas. La primera visión —-mejor conocida como la "hipótesis crisis-aprendizaje-reforma" (Stern, 1997) — sostiene que las crisis aceleran el aprendizaje político y el proceso de cambio institucional, pues sacuden las inercias y la resistencia habitual al cambio que predomina en las organizaciones gubernamentales. Un gobierno que enfrenta un episodio de crisis opera de manera muy diferente que en situaciones de normalidad: en primer lugar, la crisis tiende a saturar la atención pública, ocultando los demás asuntos que también son responsabilidad del gobierno. En segundo lugar, provoca que las acciones (e inacciones) de los actores políticos estén sujetas a un escrutinio más intenso que en situaciones de normalidad. Sin embargo, es importante tomar en cuenta que las crisis no representan una excusa para disminuir la intensidad del conflicto político, pues, por grave que resulte la situación, éste no sólo no se reduce sino que incluso se exacerba. Por todas estas características, se ha sostenido que las crisis sirven para precipitar cambios institucionales, organizacionales y de política pública que, en tiempos de normalidad, difícilmente ocurrirían.

La hipótesis "crisis-aprendizaje-reforma" ha logrado influir en diversos estudios sobre política pública, debido sobre todo a los siguientes argumentos:

comportamiento que se derivan de la experiencia y que se relacionan con la consecución o revisión de los preceptos que pertenecen a un sistema de creencias" (Sabatier, 1988: 149). 
1. Para que los tomadores de decisiones públicas pongan atención a ciertos problemas (y eventualmente los incorporen a la agenda gubernamental) se necesitan ciertos factores detonantes (focusing events). Las crisis son mecanismos que pueden servir para tal propósito (Kingdon, 1995).

2. Las situaciones de crisis fuerzan a los tomadores de decisiones a enfrentar asuntos de alta complejidad valorativa y a elegir cursos de acción que, en situaciones de normalidad, preferirían eludir. Por lo tanto, las crisis pueden desembocar en un realineamiento estratégico de las políticas (Oneal, 1982).

3. Las crisis modifican la calidad del debate público: a) Generan la expectativa de acciones contundentes, por lo que los tomadores de decisiones se ven obligados a visibilizar sus acciones (Stern, 1997). b) Como ya se comentó antes, contribuyen a la formación de coaliciones promotoras que compiten por imponer sus respectivas visiones en el debate, mejorando así el aprendizaje de políticas (Sabatier, 1988).

Existe, sin embargo, una segunda visión según la cual las situaciones de crisis dificultan el aprendizaje gubernamental. Esta perspectiva parte del supuesto de que los actores sociales tienden a responder de manera rígida e inflexible a las situaciones de riesgo que se derivan de una crisis (Staw, Sandelands y Dutton, 1981). La aparición de un evento crítico hace que el procesamiento de la información entre las agencias gubernamentales se vuelva más restringido y que los canales de comunicación entre ellas se reduzcan. En un escenario así, los tomadores de decisiones suelen acotar su atención exclusivamente a los acontecimientos que ponen en riesgo su supervivencia político-burocrática y, por lo tanto, es muy común que promuevan una centralización del poder y la influencia. Todo esto imprime una gran rigidez al proceso decisorio, cosa que en tiempos de normalidad no necesariamente ocurre.

Además de la rigidez que las crisis imprimen a las decisiones de gobierno, hay otra característica adicional que inhibe el aprendizaje: la gran politización que caracteriza a las organizaciones del sector público (Stern, 1997). Por una parte, es habitual que dichas organizaciones, en un entorno de crisis, adopten una actitud defensiva tendiente a ocultar sus fallas de desempeño, con el fin de proteger su imagen ante el público y ante otras organizaciones. Esa misma actitud defensiva ante la mirada externa ocasiona que las agencias gubernamentales sean poco proclives a tolerar que sus miembros se atrevan a emitir opiniones críticas sobre el desempeño organizacional. Esto, evidentemente, vulnera el aprendizaje. Fruto de su marcada politización es también el comportamiento oportunista que suelen 
adoptar ciertos actores organizacionales, quienes, ante un contexto de crisis, tienden a exagerar su contribución al éxito organizacional. Por último, contra la expectativa popular generalizada de que los líderes gubernamentales aprenden ciertas lecciones después de una crisis, hay evidencias que indican que los líderes suelen quedar atrapados en la "política de las culpas". Como afirman Boin y Hart:

Si en realidad existe el aprendizaje postcrisis, éste generalmente ocurre en el largo plazo. Es un proceso laborioso llevado a cabo lejos del reflector de los medios y de la turbulencia que conllevan los litigios políticos y jurídicos contra los altos líderes gubernamentales. El aprendizaje es, con frecuencia, un asunto poco heroico consistente en diseñar mejoras tecnológicas y en adaptar rutinas burocráticas. Ello requiere paciencia, memoria institucional y una atmósfera poco conflictiva - atributos que los líderes políticos no suelen tener (Boin y Hart, 2003: 548).

En síntesis, los elementos discutidos antes implican que habría que ser escépticos respecto a la capacidad de las crisis para detonar procesos de aprendizaje organizacional. En otros términos, la literatura ha dado origen a una hipótesis rival, según la cual las crisis inhiben el aprendizaje de políticas y la toma de decisiones.

Aprender de la experiencia es muy difícil en un mundo donde las brechas de desempeño son complicadas de medir; donde no hay teorías causales bien desarrolladas; donde la experimentación controlada es virtualmente imposible; donde los opositores hacen todo lo posible para enredar la situación y donde incluso los propios aliados llegan a ser recelosos debido a rivalidades personales y organizacionales (Sabatier, 1988: 151).

La siguiente sección analiza algunos de los hechos más relevantes de la crisis de la L12 del Metro de la Ciudad de México a la luz de la discusión teórica aquí esbozada, buscando sobre todo dar una respuesta preliminar a la pregunta planteada desde el inicio de este artículo: ¿qué aprendizajes pueden derivarse de dicho evento y en qué medida éstos pueden servir para propiciar cambios que mejoren la gestión de proyectos tan grandes y ambiciosos? La descripción del caso se llevó a cabo exclusivamente sobre la base de información documental. Por una parte, se hizo un seguimiento de las notas de prensa que sobre el tema aparecieron en ocho periódicos de circulación nacional desde marzo hasta agosto de 2014 (83 notas en total). ${ }^{3}$ Por la otra, se revisaron 28 documentos, la gran mayoría de ellos de carácter oficial, en los que se incluyen comparecencias de diversos actores, informes de auditorías, reportes técnicos sobre la situación de la L12 provenien-

\footnotetext{
${ }^{3}$ Los periódicos consultados fueron La Crónica, Reforma, La Jornada, Excélsior, Milenio, El Financiero, El Universal y Proceso.
} 
tes de agencias privadas y públicas, así como comunicaciones formales entre los involucrados. Si bien se intentaron llevar a cabo entrevistas con algunos involucrados, ninguno accedió a concederlas.

\section{LA CRISIS DE LA LÍNEA 12 DEL METRO DESDE LA PERSPECTIVA DEL APRENDIZAJE Y EL CAMBIO INSTITUCIONAL}

La suspensión de la línea 12 del Metro de la Ciudad de México en marzo de 2014 constituye un evento crítico por varias razones, pero fundamentalmente porque ha puesto en tela de juicio la capacidad del Gobierno del Distrito Federal para implementar con estándares de calidad razonables un servicio de transporte masivo fundamental para la vida de millones de residentes que lo usan a diario. En primer lugar, por tratarse de un proyecto que, desde su concepción y puesta en marcha, pretendió ser uno de los más emblemáticos de la administración del jefe de gobierno Marcelo Ebrard, lo cual evidentemente comprometía el capital político de un actor que buscaba contender por la presidencia de la República en el proceso electoral de 2012. En segundo lugar, por su enorme costo financiero, no sólo en lo referente a la fase de construcción, sino también en relación a los recursos que se requerían para la operación del servicio. En tercer lugar, por la gran cantidad de usuarios que la L12 dejó de atender luego de su suspensión (casi medio millón de usuarios por día). En cuarto lugar, porque su puesta en marcha involucraba a una gran cantidad de actores privados y públicos, cada uno de los cuales asumía sólo una parte de la responsabilidad de su operación. Definir con claridad la distribución de responsabilidades entre los actores no era una tarea sencilla, pues dependía de una serie de estipulaciones contractuales que, como se pudo comprobar más adelante, no fueron lo suficientemente precisas y ocasionaron una guerra de recriminaciones entre los actores. Por último, la crisis tuvo una complejidad significativa en virtud de que la implementación del proyecto trascendió los límites temporales del periodo de gobierno: mientras que la administración 2006-2012 fue principalmente responsable de su formulación y construcción —salvo por la apresurada e incompleta inauguración hecha en octubre de 2012, casi a punto de que el jefe de gobierno concluyera su mandato-, el gobierno entrante (2012-2018) fue el que en la práctica operó los servicios de la nueva línea y, apenas nueve meses después de haberse entregado definitivamente el proyecto, decidió suspender su tramo elevado por problemas de mantenimiento. ${ }^{4}$

\footnotetext{
${ }^{4}$ No es el propósito de este artículo presentar los hechos detallados de la crisis de la L12, sino únicamente enfatizar los aspectos relevantes para ilustrar mi argumento.
} 
Es importante destacar que la crisis de la L12, lejos de ser un asunto coyuntural, ha sido el resultado de una serie de transformaciones en la estructura y procesos decisorios de las organizaciones encargadas de operar el sistema de transporte colectivo creado a finales de la década de 1960, sobre todo después de la reforma al régimen político-administrativo del Distrito Federal de 1996. A partir de dicho proceso de democratización, se produjo una gran fragmentación de los actores involucrados en la creación de nuevas líneas del Metro, las decisiones se politizaron excesivamente y se fue mermando un proceso de aprendizaje institucional que había servido para guiar con relativa eficacia y estabilidad la planeación, construcción, mantenimiento y operación de diez líneas durante más de dos décadas. En otros términos, la suspensión de la L12 puede concebirse como un "desastre organizacional anunciado" que se construyó a fuerza de resquebrajar la memoria institucional adquirida durante décadas de creación de Metro. ${ }^{5}$

Lejos de tratarse de un problema de naturaleza estrictamente técnica, la crisis de la L12 constituye un asunto eminentemente político que puede constatarse en tres niveles de análisis:

1. En la conformación de dos coaliciones de actores relativamente diferenciadas que han estelarizado una batalla de acusaciones mutuas y estrategias defensivas.

2. En la manera como cada coalición ha intentado imponer su propia definición del problema de la L12 del Metro.

3. En la ambigüedad de la información sobre la crisis, mucha de la cual se ha fundamentado en las declaraciones de los propios actores involucrados, sin que haya habido una investigación independiente e imparcial que genere mayor certidumbre sobre la situación.

La decisión del Gobierno del Distrito Federal de suspender el tramo elevado de la L12 del Metro, a partir del 12 de marzo de 2014, dio origen a la conformación de dos coaliciones de actores privados y públicos, cada una de las cuales ha intentado exculparse de tener cualquier responsabilidad en el problema. La primera coalición (que en lo sucesivo denominaré "coalición defensora", por pretender justificar su participación en la etapa de la planeación, diseño y construcción de la L12) estuvo integrada por funcionarios del gobierno saliente y por una red muy diversa de actores privados encargados de su construcción, supervisión,

\footnotetext{
${ }^{5}$ Retomo esta expresión del título del trabajo de Blancas, Hernández y Arellano en este volumen.
} 
certificación y provisión de los servicios. Los principales actores públicos locales que pertenecen a esta primera coalición son el entonces jefe de gobierno, Marcelo Ebrard, así como funcionarios del Sistema de Transporte Colectivo Metro (en especial su entonces titular Francisco Bojórquez), y del organismo desconcentrado de la Secretaría de Obras y Servicios denominado Proyecto Metro (cuyo titular era Enrique Horcasitas) encargado de la construcción, equipamiento y ampliación de la red. Los actores privados que integraron esa misma coalición fueron el consorcio constructor (compuesto por las empresas ICA, Alstom Mexicana y Carso Infraestructura y Construcción) y la empresa Construcciones y Auxiliar de Ferrocarriles de México (CAF), la cual recibió el contrato para proveer y dar mantenimiento a los trenes. La segunda coalición de actores (que en lo sucesivo denominaré "coalición detractora", por ser la principal crítica del trabajo del gobierno saliente) estuvo conformada por funcionarios del gobierno entrante (entre los que destacan el jefe de gobierno Miguel Ángel Mancera y Joel Ortega, titular de sTC Metro en la administración 2012-2018), pero también por empresas privadas, algunas de las cuales formaban parte del consorcio certificador de la L12 (entre ellas ILF Consulting Engineers) y otras que fueron contratadas por el nuevo gobierno para elaborar un diagnóstico y recomendar acciones correctivas (Systra y TSO), así como por algunos integrantes de la comisión especial investigadora de la Asamblea Legislativa del DF. Debe reconocerse que esta categorización de las dos coaliciones puede ser bastante simplificadora, pues, por lo que puede deducirse de la información documental en la que se sustenta el caso, no todos sus integrantes comparten la misma perspectiva del asunto. ${ }^{6}$ Hubo, además, otros actores que también tomaron parte en la crisis de la L12, sin identificarse necesariamente con alguna de las dos coaliciones contendientes. $^{7}$

El alto grado de politización de la crisis de la L12 se manifestó también en la manera en la que cada coalición intentó imponer su definición del problema. Por una parte, el principal argumento de la coalición defensora es que al ser parcialmente inaugurada la obra el 30 de octubre de 2012, ésta contaba con el respaldo

\footnotetext{
${ }^{6}$ Por ejemplo, durante el gobierno anterior, la Secretaría de Finanzas del Gobierno del DF parecía haber cuestionado en un dictamen socioeconómico la viabilidad financiera de la tecnología férrea seleccionada para el proyecto.

${ }^{7}$ Por ejemplo, la Secretaría de la Función Pública del Gobierno Federal en su intento porque el Gobierno del DF reintegrara 489422377 pesos provenientes del Fondo Metropolitano cuyo uso para la construcción de la línea 12 no fue justificado. También la Secretaría de Hacienda y Crédito Público (sHCP) intervino en abril de 2009 para cuestionar, a través de un oficio, por qué el Gobierno del Distrito Federal había seleccionado la opción de trenes de rodadura férrea en lugar de neumática como en casi todo el sistema de transporte colectivo Metro.
} 
técnico del consorcio certificador, cuyo dictamen resultaba positivo en materia de seguridad. Además, afirmaba que los problemas de la L12 habían sido producto de su indebida operación y falta de mantenimiento preventivo y correctivo. Más aún, el ex jefe de gobierno, Marcelo Ebrard, argüía que la decisión que tomó el gobierno entrante de suspender parcialmente el servicio de la L12 no había estado debidamente fundamentada en un dictamen técnico. Por su parte, el principal argumento de la coalición detractora fue que los problemas de la L12 eran de carácter estructural, es decir que fueron ocasionados por un diseńo inadecuado durante la etapa constructiva. En especial, se señaló que la causa principal de las fallas era la incompatibilidad entre la tecnología de rodadura férrea seleccionada para el proyecto y las vías del tren, lo que ocasionaba fuertes desgastes ondulatorios en los rieles.

En síntesis, el principal punto de discrepancia entre las dos coaliciones se centró en el origen real del problema de la L12, una señalando que el problema fue estructural y la otra enfatizando que fue la falta de mantenimiento lo que causó las fallas. Como se demostraría más tarde, las fallas se debieron a múltiples causas (de planeación, diseño, construcción y operación) atribuibles a varios actores, tanto de la administración anterior como de la actual, tal como se señala en el dictamen técnico elaborado por Systra, una de las dos empresas francesas contratadas para llevar a cabo el diagnóstico y las acciones correctivas a la L12. El citado dictamen concluyó que, efectivamente, hubo incompatibilidad entre trenes y vías, pero también deficiencias en el mantenimiento y una mala gestión del problema en su conjunto.

Pero la gran politización de la crisis de la L12 tiene que ver también con el alto grado de ambigüedad en la información en la que cada coalición sustentó sus posiciones. Una de las decisiones que mayor cuestionamiento recibió por parte de la coalición promotora fue que el nuevo gobierno suspendiera el servicio en marzo de 2014 sin que existiera un dictamen técnico que sustentara tal decisión. ${ }^{8}$ Otro hecho que se suma a las dudas de la suspensión es que el director del STC Metro, Joel Ortega, miembro de la coalición detractora, en su comparecencia ante la Asamblea Legislativa del Distrito Federal (ALDF), el 21 de marzo de 2014, afirmó que las fallas se conocían desde el 8 de septiembre de 2012:

\footnotetext{
${ }^{8}$ Esa fue la principal reclamación del ex jefe de gobierno Marcelo Ebrard a la decisión de suspender el servicio. Cabe señalar, además, que en el acervo de documentos que sustentan esta investigación sobre el caso no figura ningún dictamen técnico. La única explicación dada en el aviso publicado en la Gaceta Oficial del Distrito Federal del 12 de marzo de 2014 era que la línea 12 suspendía sus servicios en algunas estaciones debido a "necesidades de diagnóstico y mantenimiento mayor".
} 
Desde el 8 de septiembre de 2012, antes de la inauguración de la línea, se detectaron desgastes ondulatorios en algunas curvas con radio menor a 300 metros. Estos desgastes han ido incrementándose, aun cuando se iniciaron trabajos de esmerilado, al grado de que el desgaste se manifiesta en todas las curvas con un radio menor de 300 metros, en aparatos de vía y aparatos de dilatación las vibraciones que se generan con el paso de los trenes se perciben muy fuertes. ${ }^{9}$

El mismo funcionario señaló en una comparecencia posterior ante la Comisión del DF de la Cámara de Diputados que el 13 de octubre de 2012 se había realizado un estudio en el que se informaba cuáles eran los puntos con problemas en la línea y cuáles operaban correctamente. En tal caso, surge la duda de por qué Ortega decidió esperar 15 meses después de su nombramiento al frente de STC Metro para suspender el servicio de la L12. Quizá no baste con haber alegado, como lo hizo en su comparecencia ante la Comisión del Distrito Federal, que su predecesor en el cargo, Francisco Bojórquez, envió el estudio citado un día después de la inauguración de la línea, es decir, el 1 de noviembre de 2012.

Por su parte, la justificación que la coalición defensora hizo de su trabajo al frente del proyecto también estuvo sustentada en información ambigua, a tal grado que sus argumentos se plasmaron en un documento anónimo titulado " $\mathrm{La}$ verdad de la línea 12 del Metro", sin que ninguna persona o institución reconociera públicamente su autoría. ${ }^{10}$

Ante la ambigüedad e insuficiencia de la información que caracterizó la crisis de la L12, no es sorprendente que los actores involucrados hayan mostrado gran recelo respecto al mecanismo elegido para dilucidar las causas del problema y sugerir acciones correctivas. En primer lugar, el consorcio constructor - uno de los actores privados al que más responsabilidad se le atribuyó por las fallas- desconoció a una de las empresas francesas - Tricaud Societé Organisé (TSO) contratada por el Gobierno del Distrito Federal para diagnosticar y corregir el problema, diciendo que se reservaba su postura respecto a las recomendaciones emitidas por ésta, ya que el consorcio constructor había propuesto — sin éxitoque se contratara a la empresa Comsa-EMTE para la reactivación de la línea $12 .{ }^{11}$ Posteriormente, la empresa CAF — la proveedora de los trenes- desconoció el dictamen de Systra, acusándolo de carecer de rigor, de estar incompleto y de estar

\footnotetext{
${ }^{9}$ Versión estenográfica de la comparecencia del Ing. Joel Ortega Cuevas, director general del Sistema de Transporte Colectivo Metro, ante la Asamblea Legislativa del Distrito Federal, el 21 de marzo de 2014.

${ }^{10}$ Dicho documento puede consultarse en la liga http://laverdadl12.mx [fecha de consulta: 1 de abril de 2015].

${ }^{11}$ La empresa Tso concluyó que la línea 12 requería un mantenimiento correctivo "pesado" e incluso recomendó un nuevo trazo de vías que implicaba, según estimaciones del propio sTC, instalar casi medio millón de piezas.
} 
"basado en apreciaciones visuales y no en pruebas científicas y cálculos justificados", lo cual, lógicamente, es la respuesta defensiva esperable de una de las coaliciones contendientes, pero que demuestra una vez más el alto grado de politización que desata una crisis. Por si esto fuera poco, uno de los diputados locales, miembro de la Comisión Investigadora de la ALDF, Cuauhtémoc Velasco, de Movimiento Ciudadano, había asegurado que existía un conflicto de intereses entre el STC Metro y las dos empresas francesas contratadas para elaborar el diagnóstico y las acciones correctivas, pues, declaró que:

Hay un clarísimo conflicto de intereses porque por un lado se cuestiona al consorcio constructor (integrado por ICA, Carso y Alstom) y por otro, se ha efectuado un entendimiento con una de las partes que es Alstom, que a su vez está asociada con tso y ésta, a su vez, con Systra. ${ }^{12}$

El proceso para el esclarecimiento de las causas y responsabilidades de la crisis de la línea 12 del Metro, al momento de escribir estas líneas, ha logrado ya ciertos avances. Como señalé antes, el informe de Systra reveló que la responsabilidad recayó en múltiples actores que intervinieron en las diversas etapas del proyecto, poniendo en grave riesgo la vida e integridad física de casi medio millón de pasajeros diarios. Además, la Contraloría General del Distrito Federal inhabilitó a 33 funcionarios de las administraciones actual y saliente (en su gran mayoría servidores del órgano desconcentrado que se creó explícitamente para construir, equipar y ampliar la red, denominado Proyecto Metro), además de imponerles sanciones económicas y, en algunos casos, denunciarlos penalmente. Sin embargo, la gran polémica que ha caracterizado al evento, así como a la información vaga y contradictoria que se generó durante su etapa más crítica, deja más incógnitas que certidumbres, por ejemplo:

1. ¿Por qué el Gobierno del DF decidió suspender el servicio sin (aparentemente) acompañar tal decisión de un dictamen técnico completo y riguroso en el que se señalaran, entre otras cosas, las consecuencias que hubiera tenido no haberlo hecho? En todo caso, si hay indicios de que la administración actual ya tenía antecedentes de las fallas, ¿por qué esperó varios meses antes de suspender el servicio?

\footnotetext{
12“Acusa diputado sobre conflicto de intereses en línea 12", El Universal, 19 de mayo de 2014, disponible en http://www.eluniversal.com.mx/ciudad-metropoli/2014/acusa-diputado-conflicto-intereses-linea-12-1011451.html [fecha de consulta: 1 de abril de 2015].
} 
2. ¿Qué papel jugó el cambio de gobierno en el destino de la línea 12? ¿La formulación o identificación del problema hubiera sido la misma si el proyecto se hubiera inaugurado mucho antes del fin del mandato de Ebrard? Realmente, como afirma la coalición defensora, ¿̨hubo alguna motivación política para decidir la suspensión?

3. ¿Por qué se creó un órgano desconcentrado especial (Proyecto Metro) para construir, equipar y ampliar la red? ¿Por qué, en su momento, las otras once líneas del sistema Metro no lo requirieron? ¿La participación del nuevo organismo en el proyecto no complicaba más su implementación? Tomando en consideración que haber dotado a Proyecto Metro de autonomía de gestión técnica, administrativa y financiera sirvió de poco para evitar que la línea 12 fallara, ¿hubo alguna otra motivación para darle tal diseño institucional?

Con tantos enigmas como los anteriores sobre la mesa, originados de un proceso altamente politizado, fragmentado en su evidencia, con actores cuyos argumentos obedecen a una simple lógica de supervivencia política y administrativa, difícilmente podríamos esperar la derivación de aprendizajes claros y útiles que sirvieran para que en el futuro se evitaran problemas como los ocurridos con la crisis de la L12 del Metro. Sin embargo, hay uno que quizá valga la pena considerar: que involucrar a tantos actores y organizaciones en el diseño e implementación de un proyecto de alcances similares al de la L12 entraña riesgos muy importantes, uno de los cuales es la dificultad de delimitar claramente las responsabilidades de cada uno en caso de que ocurran fallas, pues todos tendrán incentivos para exculparse y responsabilizar a los otros. Visto así, la recomendación más razonable sería evitar a toda costa una estrategia de implementación tan abierta e inclusiva, aunque es muy probable que ciertos tomadores de decisiones sean proclives a orientar sus proyectos en ese sentido de manera deliberada, buscando enmarañar las responsabilidades ante una eventual crisis.

\section{REFLEXIONES FINALES}

El caso de la línea 12 contrasta claramente con la hipótesis según la cual las crisis abren ventanas de oportunidad para el aprendizaje en políticas públicas, ya que las condiciones para ello nunca estuvieron presentes. En primer lugar, el objetivo central de las coaliciones que se formaron a raíz de la decisión del gobierno entrante de suspender el tramo elevado de la línea no consistía en promover algún cambio de política pública — por ejemplo en el tema de la movilidad urbana-, a partir de un conjunto de convicciones ideológicas o analíticas comunes. Por el 
contrario, su propósito primordial consistía en inculpar al grupo rival por las fallas de la L12 y eximirse de cualquier responsabilidad en el asunto con el fin de asegurar su propia supervivencia político-burocrática. De hecho, puede decirse que las dos coaliciones emergieron a la escena pública como resultado de la crisis, no antes de que ésta estallara. En segundo lugar, el choque entre ambas giró en torno a la definición del problema, es decir que cada una intentó hacer prevalecer en la opinión pública su explicación sobre las causas de que la L12 fallara. Sin embargo, el conflicto no se sustentó en evidencias sólidas, sino en desinformación, es decir en el uso sesgado y parcial de los hechos, sus causas, las decisiones y las consecuencias de éstas. Finalmente, resulta muy claro que la crisis no representó una oportunidad para el aprendizaje, sino para el posicionamiento político de los grupos en contienda, sobre todo considerando las ambiciones presidenciales de los dos jefes de gobierno: el saliente y el entrante. Por lo tanto, el caso parece robustecer más la hipótesis de que las crisis son desfavorables para el aprendizaje gubernamental, por lo menos durante el tiempo en que el asunto ocupó una gran atención mediática.

Sin duda, el caso de la L12 del Metro de la Ciudad de México representa un reto analítico que pone a prueba las herramientas convencionales del análisis de política pública. La breve descripción hecha en este artículo sobre la polémica entre las dos coaliciones, sus intentos por definir el problema para exculparse de responsabilidades y la gran ambigüedad en la información generada dan cuenta de que el caso difícilmente puede conceptualizarse como una "política pública fallida" o un clásico "problema de implementación” (Pressman y Wildavsky, 1998), en donde los tomadores de decisiones comienzan por identificar un problema, diseñar un programa o proyecto para atenderlo con base en objetivos y metas claramente definidas y, después, una vez que la política ha pasado por un periodo de ejecución, identifican que hubo una brecha entre lo planeado y lo realmente logrado para finalmente buscar explicaciones de qué falló y poner en marcha un conjunto de acciones de mejora.

El caso de la L12 tiene muy poco que ver con aquella descripción de tipo racionalista, pues nunca hubo una formulación definitiva del problema (al contrario, hubo dos definiciones que compitieron por ser reconocidas en el ámbito público, pero no con el fin de generar una solución sino para eludir la responsabilidad que cada actor pudiera haber tenido en las fallas del proyecto), su solución no tuvo una lógica única (pues por un lado se espera que el servicio de la L12 se restablezca lo antes posible bajo las mejores condiciones de seguridad para los usuarios, pero también se busca que los responsables de la crisis no queden impunes), probablemente no exista una prueba inmediata y definitiva de su solución 
(¿bastará que el consorcio constructor asuma el costo de la reparación del tramo afectado? o ¿que los responsables al más alto nivel reciban sanciones ejemplares, incluyendo las de tipo penal? o ¿̨hará falta además que haya una reestructuración institucional y organizacional en el Gobierno del DF que limite las posibilidades de que un evento similar se repita?), sus efectos son irreversibles (el terrible daño hecho a los usuarios y a la credibilidad del gobierno para operar proyectos similares difícilmente se recuperará) y la crisis de la L12 es un síntoma de otros problemas más agudos que probablemente tengan que ver con la impunidad, la corrupción, el uso del aparato gubernamental para ajustar cuentas políticas entre grupos de poder y un largo etcétera. Visto así, el caso de la L12 se parece mucho más al "problema retorcido" concebido por Rittel y Webber (1973), o bien al "fiasco de política" definido en los términos que proponen Bovens y Hart:

Un fiasco de política (policy fiasco) es un evento percibido como negativo por parte de un grupo con importancia social y política en la comunidad y que, desde la perspectiva de dicho grupo, el evento fue causado (total o parcialmente) por fallas o errores evitables y reprochables de los tomadores de decisiones de política pública. Dichas fallas pueden deberse a errores humanos por parte de los decisores, pero también a defectos institucionales o colectivos (Bovens y Hart, 2011: 15).

¿Qué aprendizajes deja esta crisis para modificar la orientación de proyectos similares en el futuro? Como lo advertí desde la introducción a este trabajo, muy pocos. Si, como afirma Sabatier (1988), el mejor aprendizaje es aquel que se deriva de la deliberación entre coaliciones promotoras opuestas, resulta evidente que la crisis de la L12 no tuvo las condiciones adecuadas para dar cauce a una discusión fundamentada en normas profesionales, pues el alto grado de politización generó que los actores se entramparan en una batalla cuyo fin primordial era inculpar al oponente y salir indemne ante la opinión pública. Hará falta que el entorno de esta crisis vea disminuida su conflictividad y que los responsables sean debidamente castigados para que los tomadores de decisiones en materia de movilidad y transporte del Gobierno del Distrito Federal puedan hacer una reconstrucción pormenorizada de los hechos y llevar a cabo los cambios institucionales, organizacionales y de política pública necesarios para lidiar de mejor manera con situaciones similares en el futuro. $\mathbf{G} \cong$ 


\section{REFERENCIAS BIBLIOGRÁFICAS}

Allison, Graham (1971), Essence of Decision: Explaining the Cuban Missile Crisis, Boston: Little Brown.

Argyris, Chris (2001), Sobre el aprendizaje organizacional, Ciudad de México: Oxford University Press.

Argyris, Chris y Donald A. Schoen (1978), Organizational Learning: A Theory of Action Perspective, Reading: Addison Wesley.

Baumgartner, Frank R., Bryan D. Jones y Peter Bjerre Mortensen (2014), "Punctuated Equilibrium Theory: Explaining Stability and Change in Policy Making", en Paul A. Sabatier y Christopher M. Weible (eds.), Theories of the Policy Process, Filadelfia: Westview Press.

Blancas, S., M. Hernández y D. Arellano (2018), “Un desastre organizacional anunciado: Dinámica organizacional e institucional en el diseño e implementación de la línea 12 del Metro en la Ciudad de México", en este volumen, pp. 125-174.

Blindenbacher, Raoul y Bidjan Nashat (2010), The Black Box of Governmental Learning: The Learning Spiral-A Concept to Organize Learning in Governments, Washington, D.C.: El Banco Mundial.

Boin, Arjen y Paul 't Hart (2003), "Public Leadership in Times of Crisis: Mission Impossible?”, Public Administration Review, 63(5), pp. 544-553.

Bovens, Mark y Paul 't Hart (2011), Understanding Policy Fiascoes, New Brunswick: Transaction Publishers.

Crozier, Michel y Erhard Friedberg (1990), El actor y el sistema: Las restricciones de la acción colectiva, Ciudad de México: Alianza Editorial Mexicana.

Etheredge, Lloyd S. (1981), "Government Learning: An Overview”, en Samuel L. Long (ed.), Handbook of Political Behavior, Nueva York: Plenum Press.

Huber, George P. (1991), "Organizational Learning: The Contributing Processes and the Literatures”, Organization Science, 2(1), pp. 88-115.

Kim, Daniel H. (1993), "The Link between Individual and Organizational Learning", Sloan Management Review, pp. 37-50.

Kingdon, John W. (1995), Agendas, Alternatives, and Public Policies, Nueva York: Harper Collins.

Majone, Giandomenico (1989), Evidence, Argument, and Persuasion in the Policy Process, New Haven: Yale University Press.

Moynihan, Donald P. (2008), "Learning under Uncertainty: Networks in Crisis Management", Public Administration Review, 68(2), pp. 350-365.

Oneal, John R. (1982), Foreign Policy Making in Times of Crisis, Columbus: Ohio State University Press. 
Pardo, M.C. y M. Vázquez (2018), “¿Un evento complejo? La historia de la puesta en marcha de la línea 12 del Sistema de Transporte Colectivo Metro en la Ciudad de México", en este volumen, pp. 19-88.

Pressman, Jeffrey L. y Aaron Wildavsky (1998), Implementación, Ciudad de México: Fondo de Cultura Económica/Colegio Nacional de Ciencias Políticas y Administración Pública.

Rittel, H.W. y M.M. Webber (1973), "Dilemmas in a General Theory of Planning”, Policy Sciences, 4(2), pp. 155-169.

Sabatier, Paul A. (1988), "An Advocacy Coalition Framework of Policy Change and the Role of Policy-Oriented Learning therein”, Policy Sciences, 21(2-3), pp. 129-168.

Simon, Hebert A. (1997), Administrative Behevior, Nueva York: The Free Press.

Staw, B.M., L.E. Sandelands y J.E. Dutton (1981), "Threat Rigidity Effects in Organizational Behavior: A Multilevel Analysis”, Administrative Science Quarterly, pp. 501-524. Stern, Eric (1997), "Crisis and Learning: A Conceptual Balance Sheet", Journal of Contingencies and Crisis Management, 5(2), pp. 69-86.

Carlos Moreno Jaimes es doctor en Políticas Públicas por la Universidad de Texas en Austin, maestro en Políticas Públicas por la Universidad de Chicago y licenciado en Administración Pública por El Colegio de México. Es profesor-investigador en el Instituto Tecnológico y de Estudios Superiores de Oriente (ITESo), donde también fue director del Departamento de Estudios Sociopolíticos y Jurídicos. Miembro del Sistema Nacional de Investigadores. Especialista en descentralización del gasto público, calidad de gobierno y evaluación del desempeño de gobiernos locales. Autor de los libros Democracia electoral y calidad gubernativa: El desempeño de los gobiernos municipales en México y Seguro Popular y federalismo en México: Un análisis de política pública (en coautoría con Laura Flamand, publicado por el cide en 2014). 